Research

Open Access

\title{
Case mix, outcome and activity for patients admitted to intensive care units requiring chronic renal dialysis: a secondary analysis of the ICNARC Case Mix Programme Database
}

\author{
Colin A Hutchison ${ }^{1}$, Alex V Crowe ${ }^{2}$, Paul E Stevens ${ }^{3}$, David A Harrison ${ }^{4}$ and Graham W Lipkin 1
}

\author{
1 University Hospital Birmingham NHS Foundation Trust, Queen Elizabeth Medical Centre, Edgbaston, Birmingham, B15 2TH, UK \\ ${ }^{2}$ Countess of Chester Hospital, Countess of Chester Health Park, Liverpool Road, Chester, Cheshire CH2 1UL, UK \\ ${ }^{3}$ Department of Renal Medicine, Kent and Canterbury Hospital, Ethelbert Road, Canterbury, Kent CT1 3NG, UK \\ ${ }^{4}$ Intensive Care National Audit \& Research Centre (ICNARC), Tavistock House, Tavistock Square, London WC1H 9HR, UK
}

Corresponding author: David A Harrison, david.harrison@icnarc.org

Received: 21 Nov 2006 Revisions requested: 3 Jan 2007 Revisions received: 8 Mar 2007 Accepted: 23 Apr 2007 Published: 23 Apr 2007

Critical Care 2007, 11:R50 (doi:10.1186/cc5785)

This article is online at: http://ccforum.com/content/11/2/R50

(C) 2007 Hutchison et al.; licensee BioMed Central Ltd.

This is an open access article distributed under the terms of the Creative Commons Attribution License (http://creativecommons.org/licenses/by/2.0), which permits unrestricted use, distribution, and reproduction in any medium, provided the original work is properly cited.

\begin{abstract}
Introduction This report describes the case mix, outcome and activity for admissions to intensive care units (ICUs) of patients who require prior chronic renal dialysis for end-stage renal failure (ESRF), and investigates the effect of case mix factors on outcome.

Methods This was a secondary analysis of a high-quality clinical database, namely the Intensive Care National Audit \& Research Centre (ICNARC) Case Mix Programme Database, which includes 276,731 admissions to 170 adult ICUs across England, Wales and Northern Ireland from 1995 to 2004.

Results During the eight year study period, $1.3 \%(n=3,420)$ of all patients admitted to ICU were receiving chronic renal dialysis before ICU admission. This represents an estimated ICU utilization of six admissions (32 bed-days) per 100 dialysis patient-years. The ESRF group was younger (mean age 57.3 years versus 59.5 years) and more likely to be male $(60.2 \%$ versus $57.9 \%$ ) than those without ESRF. Acute Physiology and Chronic Health Evaluation II score and Acute Physiology Score revealed greater severity of illness on admission in patients with ESRF (mean 24.7 versus 16.6 and 17.2 versus 12.6, respectively). Length of stay in ICU was comparable between

groups (median 1.9 days versus 1.8 days) and ICU mortality was only slightly elevated in the ESRF group (26.3\% versus $20.8 \%$ ). However, the ESRF group had protracted overall hospital stay (median 25 days versus 17 days), and increased hospital mortality (45.3\% versus $31.2 \%)$ and ICU readmission $(9.0 \%$ vs. 4.7\%). Multiple logistic regression analysis adjusted for case mix identified the increased hospital mortality to be associated with increasing age, emergency surgery and nonsurgical cases, cardiopulmonary resuscitation before ICU admission and extremes of physiological norms. The adjusted odds ratio for ultimate hospital mortality associated with chronic renal dialysis was 1.24 (95\% confidence interval 1.13 to 1.37$)$.

Conclusion Patients with ESRF admitted to UK ICUs are more likely to be male and younger, with a medical cause of admission, and to have greater severity of illness than the nonESRF population. Outcomes on the ICU were comparable between the two groups, but those patients with ESRF had greater readmission rates, prolonged post-ICU hospital stay and increased post-ICU hospital mortality. This study is by far the largest comparative outcome analysis to date in patients with ESRF admitted to the ICU. It may help to inform clinical decisionmaking and resource requirements for this patient population.
\end{abstract}

\section{Introduction}

End-stage renal failure (ESRF) is a common, chronic disorder. Advances in dialysis services over recent years have resulted in patients living increasingly independent and healthier lives. Despite this, patients with ESRF are prone to repeated hospi- tal admissions, some of which require admission to an intensive care unit (ICU). These admissions are predominantly related to the comorbidities associated with ESRF; of these, vascular access related infection and cardiovascular disease are the most common causes of admission to hospital [1].

$\overline{\mathrm{APACHE}}=$ Acute Physiology and Chronic Health Evaluation; ARF = acute renal failure; CMP = Case Mix Programme; CPR = cardiopulmonary resuscitation; ESRF = end-stage renal failure; ICNARC = Intensive Care National Audit \& Research Centre; ICU = intensive care unit; OR = odds ratio; $\mathrm{ROC}=$ receiver operating characteristic. 
A number of factors have led to a rapidly expanding ESRF population. Chief among these are issues such as increased life expectancy, resulting in the average age of the population rising, and the expanding population with predisposing chronic diseases such as diabetes mellitus [2,3]. The UK Renal Registry estimates the current incidence and prevalence of dialysis-dependent ESRF to be around 100 and 700 per million of the UK population, respectively.

Although it is perceived that the need for critical care services in the ESRF population is high and it is expected that this need will continue to increase [4], there is no adequate estimate of the actual critical care services needed. Moreover, there is no planning for critical care resource requirement to service the current ESRF population. Until recently, it was assumed that patients with ESRF admitted to critical care have considerably increased morbidity and mortality in comparison with the general ICU admission population. The recognized high ICU mortality of patients who develop acute renal failure (ARF) may in some cases be influencing the decision to admit to the ICU patients who require dialysis for ESRF. This assumption could lead to therapeutic nihilism limiting access to critical care for the ESRF population. Recently, studies including limited numbers of patients have examined this issue. Two $[5,6]$ suggested that in fact the mortality of the ESRF population in the critical care setting is only moderately raised above the nonESRF patient group, and nothing like the increased mortality seen with ARF. A third report, however, suggests that patients with ESRF in the critical care setting do have significantly increased mortality [7]. These reports also raise concerns about the predictive value of general ICU severity scoring systems to predict outcome in patients with ESRF in the critical care setting $[5,6]$.

The need for high-quality data on outcomes, and the factors that are predictive of them, in ESRF patients in the critical care setting is required to confirm or refute these previous findings. Availability of such data will help to inform service planning and guide clinical decision making in this patient population. In the present study a large, high-quality, clinical database was used to identify admissions to ICUs across England, Wales and Northern Ireland of patients with ESRF who were already receiving chronic dialysis. We report, for the first time, national, baseline information that will be useful for both local benchmarking and for dictating future policy. This report describes case mix and factors that are predictive of outcome in patients with ESRF admitted to the ICU, as a first step toward achieving the desired service goals.

\section{Materials and methods Case Mix Programme Database}

The Case Mix Programme (CMP) is a national comparative audit of adult, general critical care units in England, Wales and Northern Ireland coordinated by the Intensive Care National Audit \& Research Centre (ICNARC). Data were extracted for
276,731 admissions to 170 intensive care units (ICUs) from the CMP Database, covering the period from December 1995 to January 2004. Details of the data collection and validation were reported previously [8].

\section{Selection of cases}

Admissions were identified by the recording of the need for chronic renal replacement therapy, as part of the chronic health conditions for Acute Physiology and Chronic Health Evaluation (APACHE) II scoring [9]. The need for chronic renal replacement therapy is defined as, 'admission currently requires chronic renal replacement therapy (either chronic haemodialysis, chronic haemofiltration, or chronic peritoneal dialysis) for irreversible renal disease', and must be documented before admission or on admission to the CMP unit.

\section{Data}

Data were extracted on case mix, outcome and activity, as defined below.

\section{Case mix}

Age at admission and sex were extracted. Admissions of patients who were mechanically ventilated during the first 24 hours in the ICU were identified by recording of mechanical ventilation on admission to the unit or by recording of a lowest or highest ventilated respiratory rate during the first 24 hours after admission. The following physiological variables, selected a priori, were extracted from records of the first 24 hours in the ICU: highest serum creatinine, lowest serum albumin and lowest haematocrit.

Acute severity was measured using the APACHE II Acute Physiology Score and the APACHE II score [9]. The former encompasses a weighting for acute physiology (defined by derangement from the normal range for 12 physiological variables during the first 24 hours in the ICU). The latter additionally encompasses a weighting for age and for past medical history of specified serious conditions.

Surgical status was defined as either nonsurgical, elective surgery, or emergency surgery, based on the source of admission to the CMP unit and the National Confidential Enquiry into Perioperative Deaths (NCEPOD) classification of surgery, as was previously described [8].

Organ system failures were assessed according to the method proposed by Knaus and coworkers [10], based on physiological data from the first 24 hours in the ICU. The organ system failures assessed are cardiovascular failure, respiratory failure, renal failure, haematological failure and neurological failure. Note that all patients on chronic renal dialysis are excluded from the renal failure category, and so admissions in the study population had a possible range from zero to four organ system failures. 


\section{Outcome}

Survival data were extracted at discharge from the CMP unit and at ultimate discharge from hospital.

\section{Activity}

Length of stay in ICU was calculated in fractions of days from the dates and times of admission and discharge from the CMP unit. Length of stay in hospital was calculated in days from the dates of original admission to and ultimate discharge from an acute hospital. Transfers in from another ICU were identified as admissions whose source of admission to the CMP unit was ICU in the same or other hospital. Readmissions to ICU within the same hospital stay were identified from the postcode, date of birth and sex, and confirmed by the participating units. Treatment withdrawal was defined as the documented decision to withdraw all active treatment, other than comfort measures. The destination following discharge from the CMP unit was also extracted for all admissions of patients who were discharged alive.

\section{Analyses}

Case mix, outcome and activity were described for all patients admitted who required chronic renal dialysis and for the remainder of the CMP Database, excluding admissions of patients for whom there was no evidence available to assess past medical history. The primary reason for admission to the CMP unit (coded using the ICNARC Coding Method [11]) was tabulated for patients requiring chronic renal dialysis. Ultimate hospital mortality, by number of organ system failures, was compared for patients requiring and not requiring chronic renal dialysis.

The outcomes of patient admitted who required chronic renal dialysis, as compared with other patients, adjusted for case mix factors, were assessed with a multiple logistic regression model on ultimate hospital mortality. Case mix adjustment was performed including the following factors: age, sex, surgical status, APACHE II chronic health conditions (excluding chronic renal replacement therapy), cardiopulmonary resuscitation (CPR) during 24 hours before admission to the CMP unit, Glasgow Coma Score (lowest during the first 24 hours in the CMP unit or the pre-sedation value for patients who were sedated or paralyzed and sedated for the first 24 hours), number of organ system failures, sepsis (defined physiologically using data from the first 24 hours following admission to the CMP unit [12]) and all of the physiological variables included in the APACHE II model plus serum albumin. Age, Glasgow Coma Score and number of organ system failures were modelled as having a linear effect on the log odds. All other variables were modelled categorically, using the categories from APACHE II or APACHE III [13] as appropriate for the physiological variables, but fitting new weights to each category. When a variable was present in both APACHE II and APACHE III, the categorization giving the greatest number of categories was selected. Categories from APACHE II were used to model temperature, mean arterial pressure, arterial $\mathrm{pH}$, serum sodium, serum potassium, serum creatinine, haematocrit and white blood cell count. Categories from APACHE III were used to model heart rate, respiratory rate, oxygenation (either arterial to alveolar oxygen difference or arterial oxygen tension, depending on the fractional inspired oxygen level) and serum albumin. Patients whose records were lacking age, sex, surgical status, or any routinely measured physiological variables (temperature, blood pressure, heart rate, or respiratory rate) were excluded from the modelling. All other missing values were assumed to be normal and were placed in the category corresponding to zero APACHE II/III points.

The same multiple logistic regression approach was used to model the effects of the above parameters on ultimate hospital mortality within the group of patients requiring chronic renal dialysis. Because this involved a much smaller number of admissions, the APACHE II/III categories were first collapsed by combining adjacent categories such that each category contained at least 50 admissions. Results of this model were compared with the same model fitted in the group of patients not requiring chronic renal dialysis by introducing interaction terms.

All logistic regression models were assessed for discrimination by the area under the receiver operating characteristic (ROC) curve [14], and for overall fit by Brier's score (mean square error between outcome and prediction) [15] and Shapiro's $R$ statistic (geometric mean probability assigned to the event that occurred) [16].

The usefulness of the newly-developed ESRF-specific model in discriminating between survivors and nonsurvivors among ESRF patients and non-ESRF patients was assessed using ROC curves. The utility of the model was also compared with the performance of the APACHE II score in these groups.

All analyses were performed using Stata 8.2 (StataCorp LP, College Station, TX, USA).

\section{Results \\ Data}

Of 276,731 patients admitted to 170 adult ICUs in the CMP Database, for 270,972 (97.9\%) there was sufficient evidence to assess past medical history. Of these, 3,420 (1.3\%) were identified as requiring chronic renal dialysis. Figure 1 shows projected ICU admissions for the chronic renal dialysis population and the total population for the years of the study. In 2003, we project that there were 1,172 admissions to ICUs in England, Wales and Northern Ireland of patients requiring chronic renal dialysis, occupying a total of 5,920 ICU beddays. The UK Renal Registry Report 2004 [17] estimated the total number of adult patients receiving renal replacement therapy in 2003 in England, Wales and Northern Ireland to be 33,929 , of which $54 \%$ received dialysis. Based on these fig- 
Figure 1

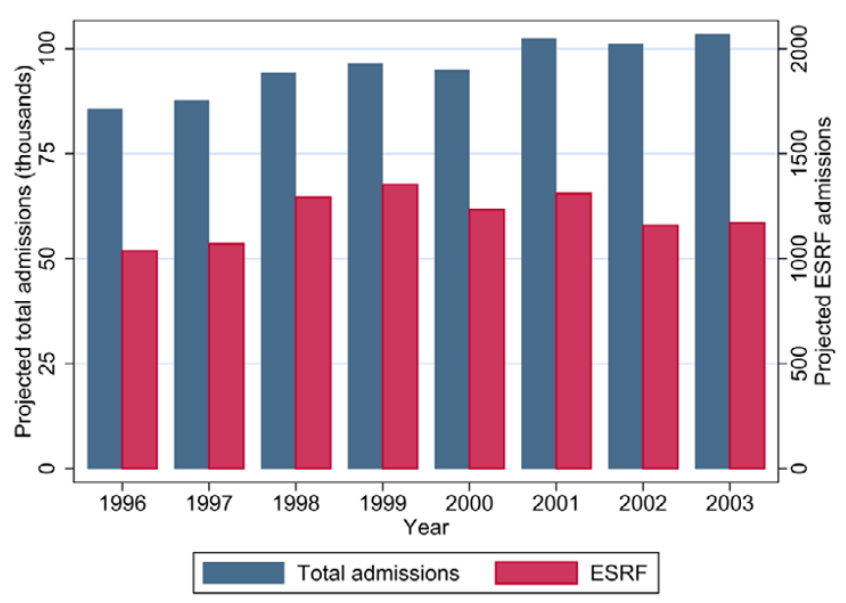

Projected total admissions to ICU and number requiring chronic renal dialysis. The figures relate to England, Wales and Northern Ireland. ESRF, end-stage renal failure (requiring chronic renal dialysis); ICU, intensive care unit.

ures, ICU utilization in 2003 was six ICU admissions or 32 ICU bed-days per 100 dialysis patients. The ICU utilization by patients with ESRF remained stable over the past five study years, whereas the numbers of patients treated nationally for ESRF increased.

\section{Case mix, outcome and activity}

Table 1 describes measures of case mix, outcome and activity for patients requiring chronic renal dialysis and admissions of all other patients for whom evidence was available to allow assessment of past medical history.

Patients requiring chronic renal dialysis were slightly younger than other patients (mean age 57.3 years versus 59.5 years) and were slightly more likely to be male $(60.2 \%$ versus $57.9 \%)$. They were more likely to have received CPR during the 24 hours before admission to the CMP unit (13.6\% versus $7.3 \%$ ). They had greater creatinine (mean $6.5 \mathrm{mg} / \mathrm{l}$ versus 1.5 $\mathrm{mg} / \mathrm{l}$ ) and lower haematocrit (mean $26.9 \%$ versus $31.3 \%$ ). Overall acute severity of illness was worse, as indicated by higher Acute Physiology Score (mean 17.2 versus 12.6) and APACHE II score (mean 24.7 versus 16.6 ). Overall, $67 \%$ of all patients requiring chronic renal dialysis were nonsurgical, as compared with $56 \%$ of other patients. The pattern of organ system failures was similar for both groups.

Crude mortality in the CMP unit was $26.3 \%$ for patients requiring chronic renal dialysis, as compared with $20.8 \%$ for other patients. At ultimate hospital discharge, mortality in these patients was $45.3 \%$ as compared with $31.2 \%$ in the reference group.

Patients requiring chronic renal dialysis had a similar length of stay in the CMP unit to that of other patients, but they had a longer stay in hospital (median 25 days versus 17 days for survivors; 15.5 days versus 8 days for nonsurvivors; Figure 2). Patients requiring chronic renal dialysis were more likely to be readmitted to the ICU during the same hospital stay (9.0\% versus $4.7 \%$ ), although the rate of direct transfers between ICUs was similar for the two groups of patients. There was no significant difference between the groups in the decision to withdraw treatment $(9.8 \%$ versus $10.7 \%$ in non-ESRF and ESRF populations, respectively). The patterns of destination following discharge were broadly similar, although patients requiring chronic renal dialysis were slightly more likely to be transferred to high dependency care and were considerably more likely to be transferred to an 'other intermediate care area', which is the category containing renal units.

Of the 3,420 patients requiring chronic renal dialysis, 3,189 (93.2\%) had a complete primary reason for admission specified, $230(6.7 \%)$ had a partially coded reason for admission, and the remaining one admission $(0.03 \%)$ had no reason for admission recorded. Of the 3,189 patients with a complete primary reason for admission, 275 (8.6\%) had chronic renal failure recorded as the reason for admission (Table 2). The most common other reasons for admission were septic shock (179 [5.6\%]) and pneumonia either with no organism isolated (167 [5.2\%]) or a bacterial pathogen isolated (94 [2.9\%]).

Hospital mortality increased steeply with number of organ system failures (Table 3). It was higher in patients requiring chronic renal dialysis, particularly at low numbers of organ system failures.

\section{Case mix adjusted effect of chronic renal dialysis on ultimate hospital mortality}

After adjusting for case mix factors of age, sex, surgical status, APACHE II physiology variables, serum albumin and the number of nonrenal organ system failures (see Materials and methods, above), the odds ratio for ultimate hospital mortality associated with chronic renal dialysis was 1.24 (95\% confidence interval $[\mathrm{Cl}] 1.13$ to 1.37$)$ as compared with a crude odds ratio before case mix adjustment of $1.82(95 \% \mathrm{Cl} 1.69$ to 1.96). The case mix adjusted model had an area under the ROC curve of 0.857 ( $95 \% \mathrm{Cl} 0.855$ to 0.858 ), a Brier's score $(B)$ of 0.138 and a Shapiro's $R$ of 0.653 when assessed for all admissions.

\section{Relationship of case mix factors with ultimate hospital mortality}

Table 4 presents the results of the multiple logistic regression analysis of case mix factors on ultimate hospital mortality in the group of chronic renal dialysis patients. The following factors were associated with increased odds of hospital mortality: older age, emergency surgery and nonsurgical cases (as compared with elective surgery), presence of other chronic health conditions, CPR during the 24 hours before admission to the CMP unit, hospital stays of longer than one week before 
Table 1

Case mix, outcome and activity for patients admitted to ICUs requiring chronic renal dialysis as compared with other patients

\begin{tabular}{|c|c|c|c|c|c|c|}
\hline \multirow[b]{2}{*}{ Case mix } & \multirow{2}{*}{$\begin{array}{l}\text { Parameter } \\
\text { Age (mean [SD]; years) }\end{array}$} & \multicolumn{2}{|c|}{$\begin{array}{l}\text { Patients requiring chronic renal } \\
\text { dialysis } \\
(n=3,420)\end{array}$} & \multicolumn{2}{|c|}{$\begin{array}{l}\text { Patients not requiring chronic renal } \\
\text { dialysis } \\
\qquad(n=267,552)\end{array}$} & \multirow{2}{*}{$\begin{array}{l}P \text { value } \\
<0.001\end{array}$} \\
\hline & & 57.3 & $(15.8)$ & 59.5 & $(19.6)$ & \\
\hline & Male sex $(n[\%])$ & 2,058 & $(60.2)$ & 154,780 & $(57.9)$ & 0.006 \\
\hline & CPR before admission $(n[\%])$ & 466 & $(13.6)$ & 19,535 & (7.3) & $<0.001$ \\
\hline & Mechanically ventilated ( $n[\%])$ & 2,107 & $(61.8)$ & 167,840 & $(63.1)$ & 0.135 \\
\hline & Highest serum creatinine (mean [SD]; mg per $100 \mathrm{ml} / \mathrm{mmol}$ per I]) & 6.5 & $(3.2) / 575$ (283) & 1.5 & $(1.3) / 133(115)$ & $<0.001$ \\
\hline & Lowest serum albumin (mean [SD]; g/l) & 22.9 & $(7.7)$ & 23.4 & (8.5) & 0.001 \\
\hline & Lowest haematocrit $(\%) /$ haemoglobin $(\mathrm{g} / \mathrm{dl})$ (mean [SD]) & 26.9 & $(5.9) / 9.0(2.0)$ & 31.3 & $(6.6) / 10.4(2.2)$ & $<0.001$ \\
\hline & APACHE II APS score ${ }^{a}$ (mean [SD]) & 17.2 & (6.5) & 12.6 & (6.7) & $<0.001$ \\
\hline & APACHE II scorea (mean [SD]) & 24.7 & $(7.0)$ & 16.6 & (7.3) & $<0.001$ \\
\hline & Surgical status $(n[\%])$ & & & & & $<0.001$ \\
\hline & Nonsurgical & 2,282 & $(66.7)$ & 150,350 & $(56.2)$ & \\
\hline & Elective surgery & 592 & $(17.3)$ & 66,017 & $(24.7)$ & \\
\hline & Emergency surgery & 545 & $(16.0)$ & 50,947 & $(19.1)$ & \\
\hline & Number of nonrenal organ system failures ${ }^{b}(n[\%])$ & & & & & $<0.001$ \\
\hline & None & 1,156 & (33.8) & 107,140 & $(40.0)$ & \\
\hline & 1 & 1,223 & $(35.8)$ & 99,299 & $(37.1)$ & \\
\hline & 2 & 743 & $(21.7)$ & 46,447 & $(17.4)$ & \\
\hline & $3+$ & 298 & $(8.7)$ & 14,666 & $(5.5)$ & \\
\hline \multirow[t]{2}{*}{ Outcome } & Mortality in ICU $(n[\%])$ & 898 & $(26.3)$ & 55,547 & $(20.8)$ & $<0.001$ \\
\hline & Ultimate hospital mortality $(n[\%])$ & 1,379 & $(45.3)$ & 77,869 & (31.2) & $<0.001$ \\
\hline \multirow[t]{19}{*}{ Activity } & ICU LOS (median [IQR]; days) & & & & & \\
\hline & Survivors & 1.9 & $(0.9-4.2)$ & 1.8 & $(0.9-4.5)$ & 0.507 \\
\hline & Nonsurvivors & 2.0 & $(0.6-6.0)$ & 1.9 & $(0.7-6.1)$ & 0.843 \\
\hline & Total hospital LOS (median [IOR]; days) & & & & & \\
\hline & Survivors & 25 & $(13-49)$ & 17 & $(9-33)$ & $<0.001$ \\
\hline & Nonsurvivors & 15.5 & $(5-35)$ & 8 & $(2-21)$ & $<0.001$ \\
\hline & Transfers from another ICU $(n[\%])$ & 120 & (3.5) & 10,508 & (3.9) & 0.210 \\
\hline & Readmissions within hospital stay ( $n[\%]$ ) & 306 & $(9.0)$ & 12,676 & $(4.7)$ & $<0.001$ \\
\hline & Treatment withdrawn $(n[\%])$ & 364 & $(10.7)$ & 26,119 & (9.8) & 0.087 \\
\hline & Destination following discharge ( $n[\%])$ & & & & & $<0.001$ \\
\hline & Ward, same hospital & 1,788 & $(70.9)$ & 155,487 & (73.4) & \\
\hline & Recovery, same hospital & 14 & $(0.6)$ & 975 & $(0.5)$ & \\
\hline & ICU, same hospital & 9 & $(0.4)$ & 1,580 & $(0.8)$ & \\
\hline & HDU, same hospital & 295 & $(11.7)$ & 28,695 & (13.6) & \\
\hline & Other intermediate care, same hospital & 156 & $(6.2)$ & 4,573 & $(2.2)$ & \\
\hline & ICU, other hospital & 94 & (3.7) & 10,898 & $(5.2)$ & \\
\hline & HDU, other hospital & 16 & $(0.6)$ & 850 & $(0.4)$ & \\
\hline & Other hospital, not ICU/HDU & 137 & $(5.4)$ & 6,088 & (2.9) & \\
\hline & Normal residence & 12 & $(0.5)$ & 2,662 & (1.3) & \\
\hline
\end{tabular}

aAcute Physiology and Chronic Health Evaluation (APACHE) II exclusions: age < 16 years; intensive care unit (ICU) stay < 8 hours; readmissions within same hospital stay; transfers from another ICU; admissions following coronary artery bypass grafting; and admissions for primary burns. bOrgan system failures assessed

physiologically according to the method of Knaus and coworkers [10]. APS, Acute Physiology Score; CPR, cardiopulmonary resuscitation; HDU, high dependency unit;

IQR, interquartile range; LOS, length of stay; SD, standard deviation. 

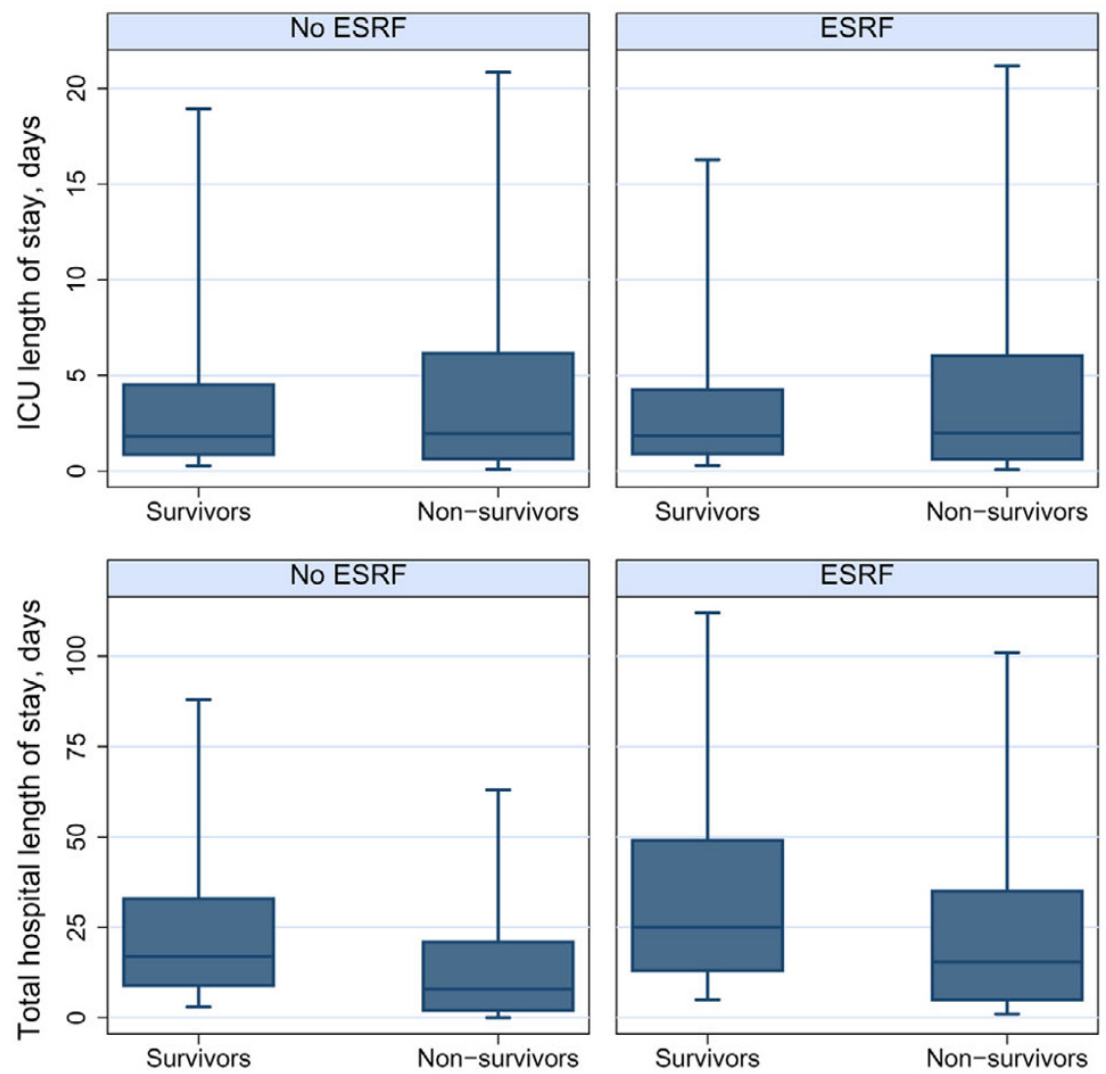

Length of stay in the ICU and in hospital. Box indicates median and quartiles; whiskers indicate 5th and 95th percentiles. ESRF, end-stage renal failure (requiring chronic renal dialysis); ICU, intensive care unit.

Table 2

\begin{tabular}{lcc}
$\begin{array}{l}\text { Most common primary reasons for admission to the ICU for } \\
\text { admissions requiring chronic renal dialysis }\end{array}$ & $n(\%)$ \\
\hline Primary reason for admission & 275 & $(8.6)$ \\
\hline Chronic renal failure & 179 & $(5.6)$ \\
Septic shock & 167 & $(5.2)$ \\
Pneumonia, no organism isolated & 94 & $(2.9)$ \\
Bacterial pneumonia & 90 & $(2.8)$ \\
Septicaemia & 87 & $(2.7)$ \\
Status epilepticus or uncontrolled seizures & 84 & $(2.6)$ \\
Cardiogenic pulmonary oedema & 84 & $(2.6)$ \\
Hypovolaemic shock & 79 & $(2.5)$ \\
Cardiogenic shock & 75 & $(2.4)$ \\
CAPD related peritonitis
\end{tabular}

CAPD, Continuous ambulatory peritoneal dialysis; ICU, intensive care unit. admission to the CMP unit, lower mean arterial pressure, high heart rate, high respiratory rate, extreme oxygenation values (high alveolar to arterial oxygen difference or low arterial oxygen tension), low arterial $\mathrm{pH}$, low serum sodium, low serum albumin, extreme (high or low) white blood count, low Glasgow Coma Score, increasing number of organ system failures, and sepsis during the first 24 hours in the CMP unit. Among patients requiring chronic renal dialysis, this model had discrimination and fit statistics as follows: area under the ROC curve $0.817(95 \% \mathrm{Cl} 0.802$ to 0.832$), B=0.173$ and $R=$ 0.595 .

When compared with the same model fitted in patients not requiring chronic renal dialysis, a number of factors exhibited a significantly different relationship with hospital mortality. Factors with a weaker association with hospital mortality in the ESRF population were age, surgical status, oxygenation, potassium and haematocrit. Adjusting for all other factors, a high mean arterial pressure ( $\geq 130 \mathrm{mmHg}$ ) appeared to exhibit a protective effect in the ESRF population, whereas in the nonESRF population it was harmful (odds ratio 0.62 versus 1.24). 
Table 3

Mortality by number of nonrenal organ system failures in patients requiring chronic renal dialysis as compared with other admissions

\begin{tabular}{ccc}
\hline \multirow{2}{*}{ Number of nonrenal organ system failures } & \multicolumn{2}{c}{ Ultimate hospital mortality (deaths/admissions [\%]) } \\
\cline { 2 - 3 } & Admissions requiring chronic renal dialysis & Admissions not requiring chronic renal dialysis \\
\hline 1 & $289 / 1,036(27.9)$ & $14,825 / 100,125(14.8)$ \\
2 & $469 / 1,077(43.5)$ & $28,071 / 92,242(30.4)$ \\
3 & $413 / 662(62.4)$ & $24,118 / 43,545(55.4)$ \\
4 & $172 / 228(75.4)$ & $9,967 / 12,907(77.2)$ \\
$888 / 997(89.1)$
\end{tabular}

\begin{abstract}
aOrgan system failures assessed physiologically, according to the method

\section{Discrimination of the APACHE II score and ESRF-} specific model

The area under the ROC curve for the APACHE II score was 0.721 (95\% Cl 0.701 to 0.741 ) for the ESRF group as compared with 0.805 (95\% Cl 0.803 to 0.807$)$ for the non-ESRF group ( $P<0.001$; Figure 3 ). This demonstrates that APACHE II scores are less sensitive in the ESRF population than in the non-ESRF population in discriminating between survivors and nonsurvivors. Discrimination was improved by using the new ESRF-specific model, but it was still worse among the ESRF group than in the non-ESRF group (area under the ROC curve 0.817 [95\% Cl 0.802 to 0.832$]$ versus $0.853[95 \% \mathrm{Cl}$ $0.851-0.854] ; P<0.001)$.
\end{abstract}

\section{Discussion}

The aim of this study was to describe the case mix and outcome of adult admissions to ICU of patients with ESRF in the UK. To our knowledge four previous studies have reported on outcomes of patients with ESRF in the ICU, three of which were single centre and all of which included relatively small populations $[5-7,18]$. These highlighted the need for a large multicentre study to describe conclusively the admission of patients with ESRF to ICUs and their outcomes. Over the examined eight-year period, $1.2 \%(n=3,420)$ of all patients admitted to the ICU $(n=276,731)$ had ESRF and were receiving dialysis (either chronic peritoneal or haemodialysis). This figure is considerably lower than the $3.7 \%$ observed in the USA [6] and $8.6 \%$ in a single centre study conducted in a French ICU [18]. This discrepancy is not surprising, given the limitations of single centre studies and the considerable differences in the utilization of both renal replacement therapy and ICU resources between different European and North American countries.

During the study period there was considerable expansion in the total number of admissions, but this was not matched by an expansion in the number of dialysis patients being admitted to ICU. This is particularly surprising because the total UK dialysis population increased by about $50 \%$ over the same time period and merits further investigation. Based on 2003 data, these figures give an annual ICU utilization of 1,172 admissions, or six admissions per 100 patients in the dialysis population. This compares to an overall ICU utilization of two admissions per 1,000 of the general population of England, Wales and Northern Ireland. It must be stressed that this utilization represents the current usage but not the need for ICU care among patients with ESRF, which is almost certainly greater and will rise as the population grows.

As seen in the study conducted by Dara and coworkers [5], admission to ICU of patients with ESRF is more common in men than women, which is consistent with the male predominance in the dialysis population. We found the ESRF population to be significantly younger than the non-ESRF population (mean age 57.3 years versus 59.5 years), which is in contrast to the work of Clermont and coworkers [6], who did not find a significant difference in age between ESRF and non-ESRF patients. This finding raises the possibility that there could be a denial of access to the ICU for the dialysis population on the basis of age. The greater serum creatinine and lower haematocrit observed in the dialysis population was not unexpected, possibly reflecting acute complications directly attributable to the underlying disease such as pulmonary oedema or hyperkalaemia.

In the present series, patients with ESRF were found to have greater severity of illness than the non-ESRF population on admission to the ICU, as defined by both the Acute Physiology Score (17.2 versus 12.6) and APACHE II score (24.7 versus $16.6)$; this is consistent with the findings of earlier studies $[6,7,18]$. This implies that ESRF patients are not being denied entry to ICU on the basis of severity of illness; rather, it raises the issue of whether late referral or acceptance of dialysis patients to ICU is influencing the findings. Some of this difference in severity of illness at admission between ESRF and non-ESRF patients could be explained by our findings that there was a significant difference in the disease aetiology between the two groups. There were significantly more nonsurgical admissions in the ESRF population (66.7\% versus $56.2 \%$ ), and a greater proportion of this group was admit- 
Critical Care Vol 11 No 2 Hutchison et al.

\section{Table 4}

Effects of age, sex, surgical status, APACHE II physiological variables, serum albumin and number of organ system failures on ultimate hospital outcome in patients requiring chronic renal dialysis

\begin{tabular}{|c|c|c|c|c|c|c|c|}
\hline \multirow[t]{2}{*}{ Parameter } & \multicolumn{5}{|c|}{ Patients requiring chronic renal dialysis } & \multicolumn{2}{|c|}{ Admissions not requiring chronic renal dialysis } \\
\hline & Deaths & $n$ & $(\%)$ & Adjusted OR (95\% Cl) & $P$ value ${ }^{e}$ & Adjusted OR (95\% Cl) & $P$ value $^{f}$ \\
\hline Age (years)* & & & & $1.28(1.20-1.36)$ & $<0.001$ & $1.50(1.49-1.52)$ & $<0.001$ \\
\hline$<45$ & 223 & 681 & (32.8) & per 10-year increase & & per 10-year increase & \\
\hline $45-54$ & 234 & 499 & $(46.9)$ & & & & \\
\hline $55-64$ & 314 & 711 & $(44.2)$ & & & & \\
\hline $65-74$ & 398 & 805 & $(49.4)$ & & & & \\
\hline $75+$ & 210 & 351 & $(59.8)$ & & & & \\
\hline Sex & & & & & 0.145 & & 0.653 \\
\hline Female & 546 & 1,220 & $(44.8)$ & Reference & & Reference & \\
\hline Male & 833 & 1,827 & $(45.6)$ & $1.15(0.95-1.38)$ & & $1.10(1.07-1.12)$ & \\
\hline Surgical status* & & & & & $<0.001$ & & $<0.001$ \\
\hline Elective surgery & 124 & 548 & $(22.6)$ & Reference & & Reference & \\
\hline Emergency surgery & 194 & 486 & (39.9) & $1.69(1.23-2.32)$ & & $2.49(2.40-2.59)$ & \\
\hline Nonsurgical & 1,061 & 2,012 & $(52.7)$ & $2.10(1.59-3.78)$ & & 3.83 (3.69-3.97) & \\
\hline Past medical history* & & & & & 0.042 & & 0.111 \\
\hline Absent & 1,121 & 2,563 & (43.7) & Reference & & Reference & \\
\hline Present & 258 & 484 & (53.3) & $1.29(1.01-1.64)$ & & $1.57(1.52-1.62)$ & \\
\hline CPR before admission* & & & & & $<0.001$ & & 0.424 \\
\hline No & 1,083 & 2,621 & $(41.3)$ & Reference & & Reference & \\
\hline Yes & 295 & 423 & $(69.7)$ & $1.90(1.44-2.52)$ & & $2.14(2.05-2.22)$ & \\
\hline LOS before admission (days)* & & & & & $<0.001$ & & 0.053 \\
\hline 0 & 378 & 863 & $(43.8)$ & Reference & & Reference & \\
\hline $1-2$ & 198 & 595 & (33.3) & $0.76(0.58-1.00)$ & & $0.99(0.96-1.02)$ & \\
\hline $2-3$ & 94 & 240 & (39.2) & $0.98(0.69-1.40)$ & & $1.09(1.04-1.14)$ & \\
\hline $3-6$ & 197 & 448 & $(44.0)$ & $0.95(0.71-1.27)$ & & $1.29(1.24-1.34)$ & \\
\hline $7+$ & 511 & 900 & $(56.8)$ & $1.95(1.52-2.49)$ & & $1.86(1.80-1.93)$ & \\
\hline Temperature $\left({ }^{\circ} \mathrm{C}\right)$ & & & & & 0.783 & & 0.078 \\
\hline$<34$ & 80 & 126 & (63.5) & $1.24(0.77-1.99)$ & & $1.96(1.84-2.08)$ & \\
\hline
\end{tabular}


Effects of age, sex, surgical status, APACHE II physiological variables, serum albumin and number of organ system failures on ultimate hospital outcome in patients requiring chronic renal dialysis

\begin{tabular}{|c|c|c|c|c|c|c|c|}
\hline $34-36$ & 456 & 946 & $(48.2)$ & $1.05(0.85-1.30)$ & & $1.23(1.19-1.26)$ & \\
\hline $36-38.5$ & 465 & 1,208 & (38.5) & Reference & & Reference & \\
\hline $38.5-39$ & 120 & 274 & $(43.8)$ & $1.16(0.85-1.59)$ & & $0.95(0.91-0.98)$ & \\
\hline$\geq 39$ & 193 & 382 & $(50.5)$ & $1.13(0.84-1.51)$ & & $1.10(1.06-1.13)$ & \\
\hline Mean arterial pressure ${ }^{a}(\mathrm{mmHg})^{*}$ & & & & & $<0.001$ & & $<0.001$ \\
\hline$<50$ & 410 & 596 & $(68.8)$ & $1.96(1.36-2.83)$ & & $1.96(1.87-2.04)$ & \\
\hline $50-70$ & 575 & 1,189 & $(48.4)$ & $1.29(0.95-1.75)$ & & $1.21(1.17-1.25)$ & \\
\hline $70-110$ & 117 & 351 & (33.3) & Reference & & Reference & \\
\hline $110-130$ & 144 & 496 & $(29.0)$ & $0.74(0.52-1.05)$ & & $0.96(0.92-1.00)$ & \\
\hline$\geq 130$ & 100 & 357 & $(28.0)$ & $0.62(0.42-0.92)$ & & $1.26(1.20-1.32)$ & \\
\hline Heart rateb $(\text { beats } / \mathrm{min})^{*}$ & & & & & $<0.001$ & & 0.638 \\
\hline$<50$ & 86 & 168 & $(51.2)$ & $1.34(0.88-2.06)$ & & $1.15(1.08-1.22)$ & \\
\hline $50-100$ & 242 & 780 & (31.0) & Reference & & Reference & \\
\hline $100-110$ & 170 & 446 & $(38.1)$ & $1.23(0.92-1.64)$ & & $1.11(1.07-1.16)$ & \\
\hline $110-120$ & 228 & 486 & $(46.9)$ & $1.57(1.18-2.08)$ & & $1.37(1.32-1.42)$ & \\
\hline $120-140$ & 373 & 697 & (53.5) & $1.81(1.40-2.36)$ & & $1.72(1.66-1.77)$ & \\
\hline $140-155$ & 137 & 241 & $(56.9)$ & $1.86(1.30-2.66)$ & & $2.15(2.06-2.24)$ & \\
\hline$\geq 155$ & 107 & 162 & $(66.0)$ & $2.09(1.35-3.23)$ & & $2.52(2.40-2.64)$ & \\
\hline Respiratory rate ${ }^{b}$ (breaths $\left./ \mathrm{min}\right)^{*}$ & & & & & $<0.001$ & & 0.082 \\
\hline$<6$ & 63 & 121 & $(52.1)$ & $0.99(0.60-1.64)$ & & $1.22(1.15-1.30)$ & \\
\hline $6-12$ & 357 & 881 & $(40.5)$ & $1.11(0.84-1.46)$ & & $1.07(1.04-1.11)$ & \\
\hline $12-14$ & 230 & 496 & $(46.4)$ & $1.38(1.01-1.88)$ & & $1.23(1.19-1.27)$ & \\
\hline $14-25$ & 207 & 503 & $(41.2)$ & Reference & & Reference & \\
\hline $25-35$ & 269 & 627 & $(42.9)$ & $1.13(0.84-1.51)$ & & $0.99(0.96-1.03)$ & \\
\hline $35-40$ & 115 & 190 & $(60.5)$ & $2.11(1.40-3.18)$ & & $1.25(1.19-1.32)$ & \\
\hline$\geq 40$ & 96 & 148 & (64.9) & $2.32(1.46-3.68)$ & & $1.49(1.41-1.56)$ & \\
\hline Oxygenation ${ }^{b}(\mathrm{mmHg})^{*}$ & & & & & 0.025 & & 0.003 \\
\hline \multicolumn{8}{|l|}{$\mathrm{A}-\mathrm{aDO}_{2}\left(\mathrm{FiO}_{2} \geq 0.5\right)$} \\
\hline$<250$ & 92 & 182 & $(50.6)$ & Reference & & Reference & \\
\hline $250-350$ & 187 & 332 & (56.3) & $1.28(0.96-1.72)$ & & $1.28(1.24-1.32)$ & \\
\hline $350-500$ & 109 & 200 & $(54.5)$ & $0.73(0.49-1.07)$ & & $1.56(1.48-1.63)$ & \\
\hline
\end{tabular}


Critical Care Vol 11 No 2 Hutchison et al.

Table 4 (Continued)

Effects of age, sex, surgical status, APACHE II physiological variables, serum albumin and number of organ system failures on ultimate hospital outcome in patients requiring chronic renal dialysis

\begin{tabular}{|c|c|c|c|c|c|c|c|}
\hline$\geq 500$ & 164 & 242 & $(67.8)$ & $1.26(0.87-1.84)$ & & $1.70(1.63-1.77)$ & \\
\hline \multicolumn{8}{|l|}{$\mathrm{PaO}_{2}\left(\mathrm{FiO}_{2}<0.5\right)$} \\
\hline$<50$ & 33 & 61 & $(54.1)$ & $1.53(0.80-2.93)$ & & $1.06(0.97-1.16)$ & \\
\hline $50-70$ & 130 & 295 & $(44.1)$ & $1.04(0.77-1.41)$ & & $0.95(0.92-0.99)$ & \\
\hline $70-80$ & 128 & 343 & (37.3) & $0.78(0.58-1.04)$ & & $0.91(0.87-0.94)$ & \\
\hline$\geq 80$ & 352 & 846 & $(41.6)$ & Reference & & Reference & \\
\hline Arterial $\mathrm{pH}^{\mathrm{a}^{*}}$ & & & & & $<0.001$ & & 0.985 \\
\hline$<7.15$ & 156 & 190 & (82.1) & $2.65(1.68-4.18)$ & & $2.95(2.78-3.13)$ & \\
\hline $7.15-7.25$ & 157 & 248 & (63.3) & $1.53(1.08-2.16)$ & & $1.65(1.59-1.72)$ & \\
\hline $7.25-7.33$ & 248 & 544 & (45.6) & $1.16(0.92-1.47)$ & & $1.20(1.16-1.23)$ & \\
\hline $7.33-7.5$ & 559 & 1,380 & $(40.5)$ & Reference & & Reference & \\
\hline$\geq 7.5$ & 79 & 153 & $(51.6)$ & $1.40(0.95-2.07)$ & & $1.40(1.33-1.48)$ & \\
\hline Serum sodiuma $(\mathrm{mmol} / \mathrm{l})^{\star}$ & & & & & 0.035 & & 0.079 \\
\hline$<130$ & 174 & 331 & (52.6) & $1.44(1.09-1.90)$ & & $1.43(1.37-1.48)$ & \\
\hline $130-150$ & 1,069 & 2,468 & (43.3) & Reference & & Reference & \\
\hline$\geq 150$ & 41 & 64 & $(64.1)$ & $1.12(0.61-2.07)$ & & $2.26(2.14-2.39)$ & \\
\hline Serum potassiuma $(\mathrm{mmol} / \mathrm{l})$ & & & & & 0.180 & & $<0.001$ \\
\hline$<3$ & 68 & 125 & $(54.4)$ & $1.15(0.74-1.78)$ & & $1.09(1.04-1.14)$ & \\
\hline $3-3.5$ & 199 & 379 & (52.5) & $1.16(0.89-1.52)$ & & $0.99(0.96-1.02)$ & \\
\hline $3.5-5.5$ & 658 & 1,482 & $(44.4)$ & Reference & & Reference & \\
\hline $5.5-6$ & 143 & 351 & $(40.7)$ & $0.91(0.68-1.21)$ & & $1.26(1.19-1.32)$ & \\
\hline $6-7$ & 145 & 370 & $(39.2)$ & $0.74(0.55-0.99)$ & & $1.39(1.31-1.48)$ & \\
\hline$\geq 7$ & 68 & 153 & (44.4) & $0.84(0.55-1.28)$ & & $1.40(1.25-1.56)$ & \\
\hline Serum creatinine $\mathrm{e}^{\mathrm{a}}(\mathrm{mg} / 100 \mathrm{ml})$ & & & & & 0.295 & & 0.095 \\
\hline$<1.5$ & 21 & 73 & $(28.8)$ & Reference & & Reference & \\
\hline $1.5-2$ & 28 & 67 & $(41.8)$ & $0.55(0.28-1.08)$ & & $1.05(1.02-1.09)$ & \\
\hline $2-3.5$ & 152 & 307 & (49.5) & $0.90(0.58-1.39)$ & & $1.37(1.32-1.42)$ & \\
\hline$\geq 3.5$ & 1,048 & 2,365 & (44.3) & $0.80(0.55-1.15)$ & & $1.25(1.18-1.32)$ & \\
\hline Serum albumin ${ }^{b}(g / l)^{*}$ & & & & & $<0.001$ & & 0.060 \\
\hline$<20$ & 453 & 823 & $(55.0)$ & $1.50(1.20-1.87)$ & & $1.37(1.34-1.41)$ & \\
\hline
\end{tabular}


Table 4 (Continued)

Effects of age, sex, surgical status, APACHE II physiological variables, serum albumin and number of organ system failures on ultimate hospital outcome in patients requiring chronic renal dialysis

\begin{tabular}{|c|c|c|c|c|c|c|c|}
\hline $20-25$ & 239 & 501 & $(47.7)$ & $1.35(1.05-1.72)$ & & $1.00(0.97-1.03)$ & \\
\hline$\geq 25$ & 350 & 990 & (35.4) & Reference & & Reference & \\
\hline Haematocrita (\%) & & & & & 0.133 & & 0.002 \\
\hline$<20$ & 164 & 324 & $(50.6)$ & $0.70(0.49-1.01)$ & & $1.26(1.18-1.34)$ & \\
\hline $20-30$ & 790 & 1,769 & $(44.7)$ & $0.86(0.70-1.06)$ & & $1.16(1.14-1.19)$ & \\
\hline$\geq 30$ & 326 & 751 & $(43.4)$ & Reference & & Reference & \\
\hline White blood count ${ }^{\mathrm{a}}\left(\times 1,000 / \mathrm{mm}^{3}\right)^{*}$ & & & & & 0.021 & & 0.884 \\
\hline$<3$ & 92 & 129 & (71.3) & $2.02(1.26-3.25)$ & & $1.70(1.60-1.79)$ & \\
\hline $3-15$ & 586 & 1,577 & $(37.2)$ & Reference & & Reference & \\
\hline $15-20$ & 252 & 533 & (47.3) & $1.05(0.83-1.33)$ & & $1.13(1.10-1.17)$ & \\
\hline $20-40$ & 287 & 508 & $(56.5)$ & $1.28(1.00-1.64)$ & & $1.26(1.22-1.30)$ & \\
\hline$\geq 40$ & 32 & 48 & $(66.7)$ & $1.34(0.67-2.70)$ & & $1.38(1.25-1.53)$ & \\
\hline Glasgow Coma Score ${ }^{*}$ & & & & $1.12(1.09-1.15)$ & $<0.001$ & 1.09 (1.09-1.09) & 0.067 \\
\hline $3-6$ & 360 & 466 & (77.3) & per decrease of 1 & & per decrease of 1 & \\
\hline $7-9$ & 73 & 138 & (52.9) & & & & \\
\hline $10-12$ & 77 & 161 & $(47.8)$ & & & & \\
\hline $13-15$ & 756 & 2,044 & (37.0) & & & & \\
\hline Organ system failures ${ }^{\mathrm{d} *}$ & & & & $1.25(1.07-1.46)$ & 0.005 & $1.31(1.28-1.33)$ & 0.561 \\
\hline 0 & 289 & 1,036 & (27.9) & per increase of 1 & & per increase of 1 & \\
\hline 1 & 469 & 1,077 & $(43.6)$ & & & & \\
\hline 2 & 413 & 662 & (62.4) & & & & \\
\hline $3+$ & 208 & 272 & (76.5) & & & & \\
\hline Sepsis* & & & & & 0.022 & & 0.060 \\
\hline No & 785 & 2,035 & (38.6) & Reference & & Reference & \\
\hline Yes & 594 & 1,012 & $(58.7)$ & $1.28(1.04-1.57)$ & & $1.04(1.02-1.07)$ & \\
\hline
\end{tabular}

A total of 2,922 patients are included in this analysis. Significant factors $(P<0.05)$ in the model for admissions of patients requiring chronic renal dialysis are highlighted by asterisks. Exclusions are as follows: readmissions within the same hospital stay; and admissions missing age, sex, surgical status, temperature, mean arterial pressure, heart rate or respiratory rate. Those admissions with missing values for any other physiological variables were assumed to be normal and placed in the reference category. aCategories from Acute Physiology and Chronic Health Evaluation (APACHE) II (reference category equivalent to zero APACHE II points).

bCategories from APACHE III (reference category equivalent to zero APACHE III points). cPre-sedation values used for admissions sedated or paralyzed during the first 24 hours in intensive care unit (ICU). dOrgan system failures assessed physiologically, in accordance with the method of Knaus and coworkers [10]. $P^{P}$ value for significance of factor in the end-stage renal failure (ESRF) model. ${ }^{f} P$ value for difference between ESRF and non-ESRF models (test of interaction). A-aDO ${ }_{2}$, alveolararterial oxygen difference; $\mathrm{Cl}$, confidence interval; CPR, cardiopulmonary resuscitation; $\mathrm{FiO}_{2}$, fractional inspired oxygen; $\mathrm{LOS}$, length of stay; OR, odds ratio; $\mathrm{PaO}{ }_{2}$, arterial oxygen tension. 

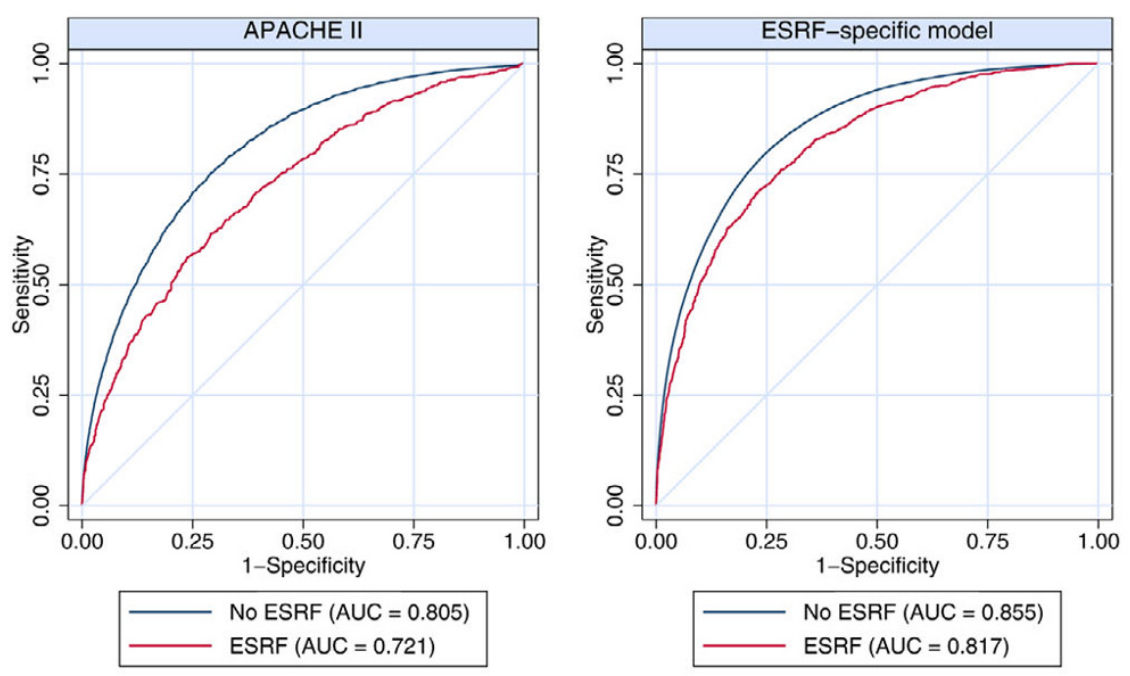

Comparison between APACHE II and the ESRF-specific model in discriminating between survivors and nonsurvivors. Shown are receiver operating characteristic curves for APACHE II and the ESRF-specific model from Table 4 for patients admitted to intensive care units requiring and not requiring chronic renal dialysis. APACHE, Acute Physiology and Chronic Health Evaluation; ESRF, end stage renal failure (requiring chronic renal dialysis).

ted following CPR. It is not possible from our analysis to determine whether the ESRF group who required pre-admission CPR suffered a primary cardiac arrhythmia, the incidence of which is known to be increased in this patient population. The admission cause was coded as chronic renal failure in a small percentage of the ESRF cohort. This probably reflects a direct acute complication of renal failure such as volume overload or electrolyte disturbance.

Somewhat surprisingly, we found that the length of ICU stay was equivalent between the two groups of patients, despite the ESRF patients being sicker and having greater comorbidity. Many UK renal units have considerable experience in the care of critically ill patients with renal disease, thus allowing earlier ICU discharge. This finding is consistent with the findings of Clermont and coworkers [6]. However, the ESRF population had a significantly longer hospital stay following discharge from ICU ( 25 days versus 17 days in the non-ESRF group for hospital survivors). There was also a marked difference in readmission rates between the two groups $(9.0 \%$ in ESRF patients versus $4.7 \%$ in non-ESRF patients). These figures could provide a basis for estimating the minimum service required to provide ICU services for the ESRF population and the costs of this service.

The large numbers included in the present study enabled us to achieve statistically significant confirmation of the suggestions made by Clermont [6] and Dara [5] and their coworkers, namely that the increased mortality observed in the ESRF population in the ICU setting is significantly lower than the increased mortality seen with ARF in the ICU setting. We found that the mortality at discharge from the ICU was 5.5\% higher in the ESRF population (26.3\% versus $20.8 \%$ in the
non-ESRF population). This increased ICU mortality probably reflects the increased severity of illness seen in this population. However, the mortality rate was considerably lower than that seen in a contemporaneous population of patients for whom ARF was recorded as the primary cause of ICU admission in an analysis of the same database (43.3\%) [19]. Although increased mortality is seen in the ESRF population admitted to ICU, the mortality rate is considerably lower than that seen in the ARF population.

The ultimate hospital mortality in the ESRF population was $45.3 \%$ (95\% Cl $43.5 \%$ to $47.0 \%)$, as compared with $31.2 \%$ $(95 \% \mathrm{Cl} 31.0 \%$ to $31.4 \%)$ in the non-ESRF population. Although this represents a significantly increased mortality in the ESRF group, it once again is much lower than the ultimate hospital mortality seen in the population of patients with ARF as their primary reason for ICU admission in the same dataset (58.6\%) [19]. This difference is even more marked when one examines subgroups of the ARF population, such as those patients presenting with oliguric ARF to the ICU, who have an ultimate hospital mortality of 70.3\% [19]. When examining outcomes for number of organ system failures, we found the ESRF population to have a higher mortality with a lower number of nonrenal organ failures.

We demonstrated significant differences in outcome between the two groups in terms of increased hospital stay following discharge from the ICU, increased rate of readmission to ICU and increased ultimate hospital mortality. It is likely that there are multiple reasons for these differences in outcome between the two groups. These include issues such as lack of physiological reserve in the dialysis population, and the well described increased rates of hospital-acquired infections in 
the ESRF population, including a high rate of staphylococcal bacteraemia and Clostridium difficile infection [20-22]. There is also the ongoing need for vascular and peritoneal access, with their corresponding complications; all of these probably contribute to these differences in outcome. Drawing from these significant differences in outcome, it may be that current management of the ESRF population following ICU discharge is suboptimal in England, Wales and Northern Ireland. There may be a greater need for closer post-ICU monitoring and increased focus on intermediate care, including rehabilitation. This requires further investigation but it does raise the possibility that, with adequate resource planning and delivery, it may well be possible to reduce some of these differences.

Analysis of factors that affect outcome in the ESRF group revealed no real surprises. Significant factors were older age; surgical status; physiological extremes such as hypotension, bradycardia, tachypnoea and hypoxia; biochemical derangement with hyponatraemia, sepsis and leucopenia; and the number of additional nonrenal organ system failures. A number of the factors examined exhibited either a stronger or weaker relationship to outcome in the ESRF population than in the non-ESRF population.

Analysis of ROC curves demonstrated that the APACHE II score's discrimination of patient outcome in the ESRF population was worse than that among patients admitted to ICU who did not require dialysis. This is consistent with previous work in patients with ARF admitted to the ICU. Lins and coworkers [23] demonstrated that the APACHE II score is a less sensitive predictor of outcome in the ARF setting than are renalspecific scoring systems such as the Stuivenberg Hospital Acute Renal Failure system [23]. This should be considered in any clinical decision-making process in patients with ESRF being considered for admission to the ICU.

\section{Conclusion}

Patients with dialysis-dependent ESRF who are admitted to UK ICUs are more likely to be younger and male, with a medical cause of admission, and to have greater severity of illness than the non-ESRF population. Despite this, ICU stay was similar and ICU mortality for patients with ESRF was only marginally increased. Nevertheless, patients with ESRF had increased ICU readmission rates, prolonged hospital stay and greater post-ICU mortality as compared with the general ICU population. Patient outcomes were considerably better than those reported for patients with ARF admitted to the ICU. This report may facilitate planning of adequate ICU resources for this population. It may also inform the clinical decision process surrounding ICU admission for patients receiving chronic dialysis therapy.

\section{Competing interests}

The authors declare that they have no competing interests.

\section{Key messages}

- ICU utilization by dialysis patients in the UK is approximately six admissions (32 bed-days) per 100 patients per year.

- Patients with ESRF admitted to ICU are more likely to be younger and male, with a medical cause for admission, and to have a greater severity of illness than are non-ESRF patients.

- Patients with ESRF have increased ICU readmission rates, prolonged hospital stay and increased post-ICU mortality (adjusted odds ratio 1.24 as compared with non-ESRF patients); however, mortality is considerably lower than for those patients with ARF who require ICU admission.

- The APACHE II score has lower sensitivity in discriminating between survivors and non-survivors in the ESRF population than in the non-ESRF population.

- These data allow, for the first time, estimation of minimum utilization of ICU services by the ESRF population in the UK and should facilitate service planning.

\section{Authors' contributions}

AVC, PES, DAH and GWL designed the study. DAH performed the analyses. $\mathrm{CAH}, \mathrm{DAH}$ and $\mathrm{GWL}$ drafted the manuscript. All authors contributed to the interpretation of results and critical revision of the manuscript, and have read and approved the final manuscript.

\section{Acknowledgements}

This study was supported by ICNARC. The authors wish to thank everyone in the ICUs participating in the CMP [24]. We acknowledge the Department of Health and the Welsh Health Common Services Authority for the initial, two-year, pump-priming funds in 1994 to establish ICNARC.

\section{References}

1. Sarnak MJ, Levey AS: Epidemiology, diagnosis, and management of cardiac disease in chronic renal disease. I Thromb Thrombolysis 2000, 10:169-180.

2. Stengel B, Billon S, Van Dijk PC, Jager KJ, Dekker FW, Simpson K, Briggs JD: Trends in the incidence of renal replacement therapy for end-stage renal disease in Europe, 1990-1999. Nephrol Dial Transplant 2003, 18:1824-1833.

3. Roderick P, Armitage A: Renal services for people with diabetes in the UK. Diabet Med 2002, 19 Suppl 4:56-60.

4. Schetz M: Renal replacement therapy in patients with chronic (end stage) renal failure and acute illness. In Critical Care Nephrology Edited by: Ronco C, Bellomo R. Boston, MA: Kluwer Academic Publishers; 1998:1139-1153.

5. Dara SI, Afessa B, Bajwa AA, Albright RC: Outcome of patients with end-stage renal disease admitted to the intensive care unit. Mayo Clin Proc 2004, 79:1385-1390.

6. Clermont G, Acker CG, Angus DC, Sirio CA, Pinsky MR, Johnson JP: Renal failure in the ICU: comparison of the impact of acute renal failure and end-stage renal disease on ICU outcomes. Kidney Int 2002, 62:986-996.

7. Uchino S, Morimatsu H, Bellomo R, Silvester W, Cole L: Endstage renal failure patients requiring renal replacement therapy in the intensive care unit: incidence, clinical features, and outcome. Blood Purif 2003, 21:170-175. 
8. Harrison DA, Brady AR, Rowan K: Case mix, outcome and length of stay for admissions to adult, general critical care units in England, Wales and Northern Ireland: the Intensive Care National Audit \& Research Centre Case Mix Programme Database. Crit Care 2004, 8:R99-R111.

9. Knaus WA, Draper EA, Wagner DP, Zimmerman JE: APACHE II: a severity of disease classification system. Crit Care Med 1985, 13:818-829.

10. Knaus WA, Draper EA, Wagner DP, Zimmerman JE: Prognosis in acute organ-system failure. Ann Surg 1985, 202:685-693.

11. Young JD, Goldfrad C, Rowan K: Development and testing of a hierarchical method to code the reason for admission to intensive care units: the ICNARC Coding Method. $\mathrm{Br} J$ Anaesth 2001, 87:543-548.

12. Padkin A, Goldfrad C, Brady AR, Young D, Black N, Rowan K: Epidemiology of severe sepsis occurring in the first 24 hours in intensive care units in England, Wales and Northern Ireland. Crit Care Med 2003, 31:2332-2338.

13. Knaus WA, Wagner DP, Draper EA, Zimmerman JE, Bergner M, Bastos PG, Sirio CA, Murphy DJ, Lotring T, Damiano A, Harrell FE Jr: The APACHE III prognostic system. Risk prediction of hospital mortality for critically ill hospitalised adults. Chest 1991, 100:1619-1636.

14. Hanley JA, McNeil BJ: The meaning and use of the area under a receiver operating characteristic (ROC) curve. Radiology 1982, 143:29-36.

15. Brier GW: Verification of forecasts expressed in terms of probability. Monthly Weather Review 1950, 75:1-3.

16. Shapiro AR: The evaluation of clinical predictions. $N$ Engl J Med 1977, 296:1509-1514.

17. The UK Renal Registry: The Seventh Annual Report 2004 [http:// www.renalreg.com/Report\%202004/Cover Frame.htm]. Bristol: UK Renal Registry

18. Manhes G, Heng AE, Aublet-Cuvelier B, Gazuy N, Deteix P, Souweine $\mathrm{B}$ : Clinical features and outcome of chronic dialysis patients admitted to an intensive care unit. Nephrol Dial Transplant 2005, 20:1127-1133.

19. Stevens $P$, Crowe $A$, Lipkin $G$, Harrison $D$ : The changing face of ARF in the intensive care unit: data from the Casemix Programme [abstract]. Proceedings of the XLII ERA-EDTA Congress; 4-7 June 2005; Istanbul, Turkey 2005 [http:// www.abstracts2view.com/era05/]. Parma, Italy: European Renal Association

20. Kausz $A$, Pahari $D$ : The value of vaccination in chronic kidney disease. Semin Dial 2004, 17:9-11.

21. Bloembergen WE, Port FK: Epidemiological perspective on infections in chronic dialysis patients. Adv Ren Replace Ther 1996, 3:201-207.

22. Cunney RJ, Magee C, McNamara E, Smyth EG, Walshe J: Clostridium difficile colitis associated with chronic renal failure. Nephrol Dial Transplant 1998, 13:2842-2846.

23. Lins RL, Elseviers MM, Daelemans R, Arnouts P, Billiouw JM, Couttenye M, Gheuens E, Rogiers P, Rutsaert R, Van der Niepen P, et al: Re-evaluation and modification of the Stuivenberg Hospital Acute Renal Failure (SHARF) scoring system for the prognosis of acute renal failure: an independent multicentre, prospective study. Nephrol Dial Transplant 2004, 19:2282-2288.

24. Participating units [ICNARC-Intensive Care National Audit \& Research Centre] [http://www.icnarc.org/audit/cmp/participat ing-units] 